\title{
Scattering amplitude of a single fracture under uniaxial stress
}

\section{Journal Article}

Author(s):

Blum, Thomas E.; Wijk, Kasper van; Snieder, Roel

Publication date:

2014-05

Permanent link:

https://doi.org/10.3929/ethz-b-000083669

Rights / license:

In Copyright - Non-Commercial Use Permitted

Originally published in:

Geophysical Journal International 197(2), https://doi.org/10.1093/gji/ggu039 


\title{
Scattering amplitude of a single fracture under uniaxial stress
}

\author{
T.E. Blum, ${ }^{1, *}$ K. van Wijk ${ }^{2}$ and R. Snieder ${ }^{3}$ \\ ${ }^{1}$ Department of Geosciences, Boise State University, Boise, ID 83725, USA. E-mail: thomas.blum@erdw.ethz.ch \\ ${ }^{2}$ Physical Acoustics Laboratory, Department of Physics, The University of Auckland, Private Bag 92019, Auckland, New Zealand \\ ${ }^{3}$ Center for Wave Phenomena Colorado School of Mines, Golden, CO 80401, USA
}

Accepted 2014 January 29. Received 2014 January 29; in original form 2013 May 22

\begin{abstract}
SUMMAR Y
Remotely sensing the properties of fractures has applications ranging from exploration geophysics to hazard monitoring. Newly developed capabilities to measure the in-plane component of dense laser-based ultrasound wave fields allow us to test the applicability of a linear slip model to describe fracture properties. In particular, we estimate the diameter, and the normal and tangential compliance of a fracture from the measured scattering amplitudes of $P$ and $S$ waves in the laboratory. Finally, we show that the normal compliance decreases linearly with increasing uniaxial static stress in the plane of the fracture, but that our measurements of the $S V$ scattered field do not show significant changes in the tangential compliance.
\end{abstract}

Key words: Microstructures; Fracture and flow; Wave scattering and diffraction.

\section{INTRODUCTION}

Fully characterizing a fracture assuming linear slip behaviour involves estimating both the normal and tangential components of the compliance. In exploration geophysics, the ratio between normal and tangential compliance is used as a proxy for the presence of fluids in the fracture (Hudson et al. 1997; Liu et al. 2000; Lubbe et al. 2008).

Hydrocarbon reservoirs or aquifers are subjected to changes in the local stress as a result of production. Time-lapse monitoring of stress through changes in fracture properties can help assess reservoir conditions. Similarly, in volcanic environments, the stress is related to volcanic activity, and dykes, local fractures, as well as the volcanic conduit all respond to changes in stress (Gudmundsson 2006).

For multiple sets of parallel fractures of a small size compared to the dominant wavelength, wave propagation can be expressed in terms of effective medium theory, widely covered in existing work (Crampin 1981; Hudson 1981; Schoenberg \& Douma 1988; Schoenberg \& Sayers 1995; Kachanov \& Sevostianov 2005). Conversely, the opposite case where the fracture plane is infinite leads to frequency-dependent reflection and transmission coefficients (Pyrak-Nolte et al. 1990a; Pyrak-Nolte \& Nolte 1992; Zhu $\&$ Snieder 2002).

Based on the linear slip model for a dry fracture, we derived the scattering amplitude in the frequency domain under the Born approximation for all combinations of incident and scattered wave modes, without making assumptions about the fracture size or wavelength, which can therefore be applied for a fracture of arbitrary size

\footnotetext{
* Now at: The Institute of Geophysics, Department of Earth Sciences, ETH Zurich, 8092 Zurich, Switzerland.
}

(Blum et al. 2011). Laser-based ultrasonic laboratory measurements of the $P$ wave scattered by a single fracture in clear plastic allowed us to quantify the normal compliance of a fracture, but were much less sensitive to the tangential component of compliance. With the development of a laser-based receiver that can measure the in-plane component of the wavefield (Blum et al. 2010), we include scattered shear wave modes, and show that these are sensitive to the shear compliance of the fracture. After independently estimating both the normal and tangential component of the compliance, we conclude by exploring the stress dependence of these fracture properties in the laboratory. However, before we introduce the experimental data, we highlight the main theoretical results of Blum et al. (2011).

\section{THEORETICAL BACKGROUND}

We consider a single homogeneous fracture following the linear slip model (Schoenberg 1980), and assume that the slip discontinuity is related to the traction $\mathbf{T}$ at the fracture by a compliance matrix $\eta$ that can be further decomposed in normal and tangential components $\eta_{N}$ and $\eta_{T}$, respectively (see Blum et al. 2011, for more details). The fracture as a whole is treated as a scatterer under the Born approximation. The $P-P$ scattering amplitude of plane waves by a such fracture can be expressed in the frequency domain as

$$
\begin{aligned}
& f_{P, P}(\hat{\mathbf{n}} ; \hat{\mathbf{m}})=\frac{\omega^{2}}{4 \pi \rho \alpha^{4}} A F\left(k_{\alpha}(\hat{\mathbf{n}}-\hat{\mathbf{m}})\right) \\
& \quad \times\left\{(\lambda+\mu)^{2} \eta_{N}+(\lambda+\mu) \mu \eta_{N}(\cos 2 \psi+\cos 2 \theta)\right. \\
& \left.\quad+\mu^{2} \eta_{N} \cos 2 \psi \cos 2 \theta+\mu^{2} \eta_{T} \sin 2 \psi \sin 2 \theta \cos \varphi\right\},
\end{aligned}
$$

where $\omega$ is the angular frequency, $\alpha$ the $P$-wave velocity and $\rho$ the density of the material, $\lambda$ and $\mu$ the Lamé parameters, $A$ the area of the fracture and $k$ the wavenumber. The angles $\psi, \varphi$ and $\theta$ and the 


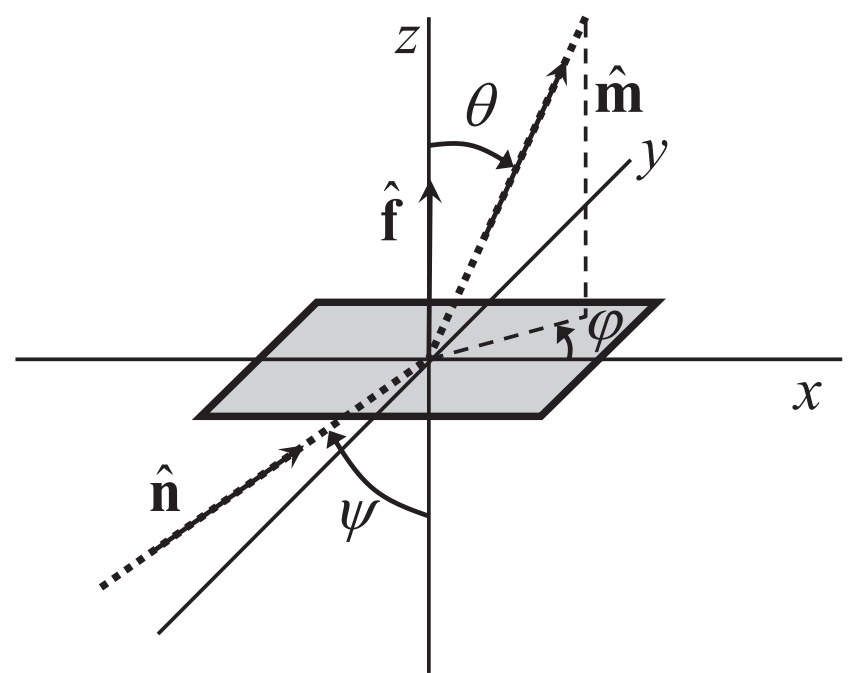

Figure 1. Definition of angles for incoming and outgoing waves from a fracture (shaded area).

unit vectors $\hat{\mathbf{n}}$ and $\hat{\mathbf{m}}$ of the direction of the incoming and outgoing waves are defined in Fig. 1.

The form factor $F$ depends on the fracture size and shape, but in the case of a circular fracture, it can be expressed as (eq. 33 of Blum et al. 2011):

$F(\mathbf{k})=\frac{2}{k_{\|} a} J_{1}\left(k_{\|} a\right)$,

where $a$ is the radius of the fracture, $k_{\|}$the projection of the wavenumber change during scattering onto the fracture plane and $J_{1}$ the Bessel function of order 1 . This derivation does not rely on assumptions about the size of the fracture with respect to the wavelength. For this work, we consider the case of a fracture which size, quantified by its radius $a$, is on the order of the elastic wavelength $\lambda$.

Under the same conditions and assumptions, the $S V$ to $S V$ scattered amplitude is

$$
\begin{aligned}
& f_{S V, S V}(\hat{\mathbf{n}}, \hat{\mathbf{p}} ; \hat{\mathbf{m}}, \hat{\mathbf{q}})=\frac{\omega^{2}}{4 \pi \rho \beta^{4}} A F\left[k_{\beta}(\hat{\mathbf{n}}-\hat{\mathbf{m}})\right] \\
& \quad \times\left\{\mu^{2} \eta_{N} \sin 2 \psi \sin 2 \theta+\mu^{2} \eta_{T} \cos 2 \psi \cos 2 \theta \cos \varphi\right\},
\end{aligned}
$$

where $\beta$ is the $S$-wave velocity and $\hat{\mathbf{p}}$ and $\hat{\mathbf{q}}$ the incoming and outgoing polarization unit vectors, respectively. The orientation of the vectors is shown in Fig. 2, the SV polarization is defined with respect to the fracture plane.

Eq. (1) shows that the $P$ to $P$ scattering is strongest and dependent on $\eta_{N}$ only when the incoming $P$ wave is normal to the plane of the fracture $\left(\psi=0^{\circ}\right)$, for an outgoing wave also normal to the fracture, either $\theta=0^{\circ}$ (forward scattering) or $\theta=180^{\circ}$. Similarly, eq. (3) shows that the $S V$ to $S V$ scattering is also most dependent on $\eta_{T}$ only for the same geometry. In the following, we consider the backscattering only, because the forward scattered wave interferes with the direct wave.

\section{EXPERIMENTAL SETUP}

We create a single disc-shaped fracture by focusing a high-power $Q$-switched Nd:YAG laser in a cylinder made of cast Poly(methyl methacrylate) (PMMA), with a diameter of $50.8 \mathrm{~mm}$ and a height of $150 \mathrm{~mm}$. The laser generates a short pulse $(\sim 10 \mathrm{~ns})$ of infrared (IR) light that is absorbed by the sample material at the focal point

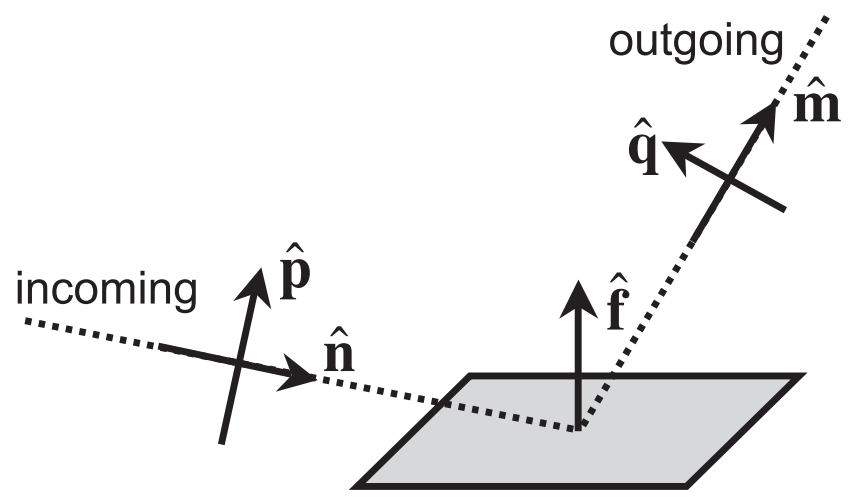

Figure 2. Definition of the normal vector $\hat{\mathbf{f}}$ to the fracture (shaded), the directions $\hat{\mathbf{n}}$ and $\hat{\mathbf{m}}$ of the incoming wave and outgoing waves, respectively. These vectors are also the polarization vectors in case of $P$ waves. For $S V$ waves, the polarization vectors of incoming and outgoing waves are $\hat{\mathbf{p}}$ and $\hat{\mathbf{q}}$, respectively.

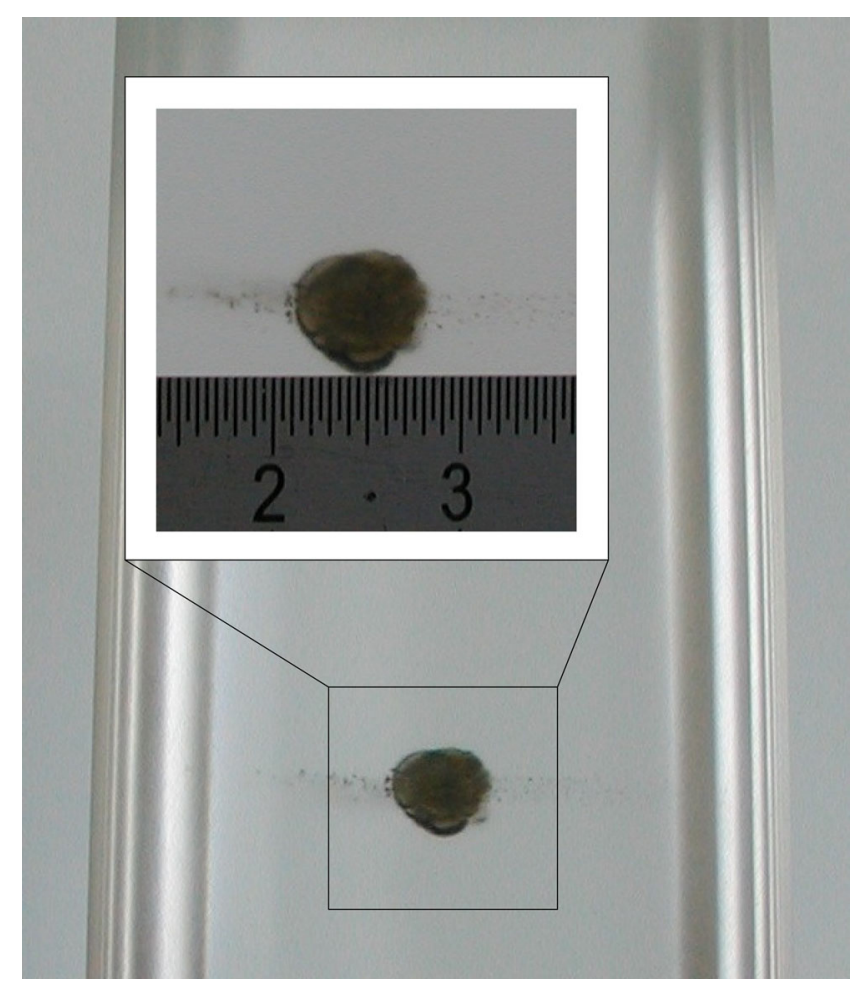

Figure 3. Photograph of the laboratory sample and zoom around the diskshaped fracture, with ruler units in centimetres. The sample is cut in half longitudinally to display the fracture without optical deformation by the curvature of the sample. The radius of the fracture is $\sim 3.5 \mathrm{~mm}$, and the diameter of the cylinder is $50.8 \mathrm{~mm}$. Small 'bubbles' on the sides of the fracture were created during a second set of experiments after the work presented here and therefore do not have any effect on the measurements.

and converted into heat. The sudden thermal expansion generates sufficient stress to form a fracture inside the plastic material (Zadler \& Scales 2008; Blum et al. 2011). Anisotropy in the elastic moduli, caused by the fabrication process, results in a fracture oriented along the cylindrical axis. The fracture studied here is approximately circular with a radius of $\sim 3.5 \mathrm{~mm}$ (Fig. 3).

Elastic waves are excited at the surface of the sample by using the same high-power $Q$-switched Nd:YAG laser, operated at a much lower power, and with a beam partially focused on the surface 


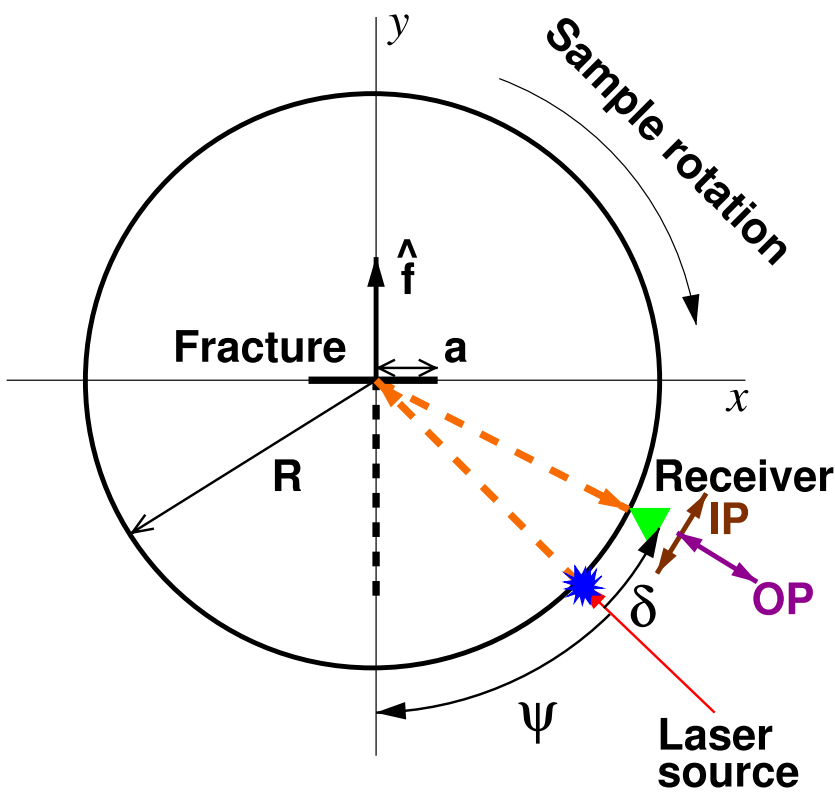

Figure 4. Schematic of the experimental setup. The source-receiver angle is fixed and the fracture rotates in respect to both source and receiver. The direction of propagation is marked with the orange dashed line. OP and IP indicate the polarization of the out-of-plane and in-plane components, respectively.

of the cylinder. When an energy pulse from the laser strikes an optically absorbing surface, part of that energy is absorbed and converted into heat. The resulting localized heating causes thermal expansion, which, in turn, results in elastic waves in the ultrasonic range (Scruby \& Drain 1990). Such a thermoelastic source generates waves over a wide range of frequencies, depending on material properties. In this experiment, most of the elastic energy is in the $200 \mathrm{kHz}-5 \mathrm{MHz}$ range.

We measure the elastic displacement with a laser interferometer. Our adaptive laser ultrasonic receiver is based on a doubled $\mathrm{Nd}$ :YAG laser, generating a constant wave (CW) $250 \mathrm{~mW}$ beam at a wavelength of $532 \mathrm{~nm}$. The receiver uses two-wave mixing in a photorefractive crystal to deliver the displacement of the sample surface. This receiver measures the out-of-plane (vertical) as well as one in-plane (horizontal) component of the displacement field. It is calibrated to output the absolute displacement field in nanometres. (See Blum et al. 2010, for a complete description). The frequency response of the receiver is flat between $20 \mathrm{kHz}$ and $20 \mathrm{MHz}$, and it can accurately detect displacements on the order of parts of an ångström.

Since the cylinder is transparent for both IR and green light, we apply aluminium tape to the surface. The tape plays the role of the absorbing medium on the source side, and reflects light back for a wide range of angles to the laser receiver, allowing the measurement of both out-of-plane and in-plane components.

The cylindrical PMMA sample is mounted on a rotational stage, whereas the locations of the non-contacting ultrasonic source and receiver are fixed in the laboratory frame of reference. The sourcereceiver angle $\delta$ (defined in Fig. 4) is therefore constant, and only the orientation of the fracture with respect to the frame of reference, characterized by the angle $\psi$ between the normal to the fracture and direction of the incoming wave, changes. We choose to fix $\delta=20^{\circ}$, which gives us the maximum backscattered amplitude, within the limitations of the experimental setup. Moreover, the source and receiver are focused on the sample in an $(x, y)$ plane normal to

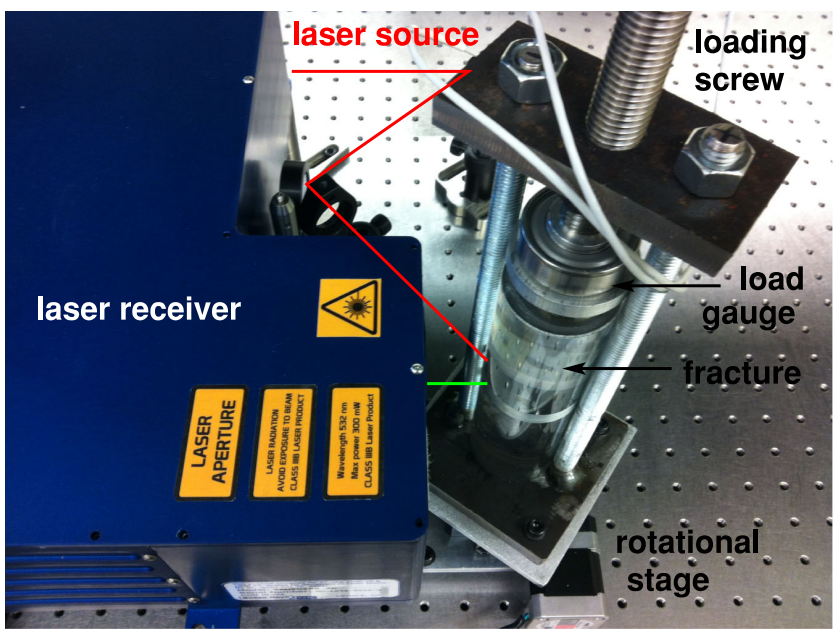

Figure 5. Photograph of the laboratory setup, including the source laser beam, laser receiver, load gauge above the sample and the load screw on top of the assembly.

the cylinder axis ( $z$-axis, Fig. 4). While anisotropic, as mentioned above, the extruded PMMA is transversely isotropic, and its elastic properties are therefore invariant with respect to the defined angles of interest.

In order to put the sample under static stress for the second part of this study, we load it by tightening a screw pushing the top of the cylinder down. We use a bearing to accommodate the rotation of the loading screw, and insert a load gauge in between the bearing and the sample to measure the compressional stress. A picture of the laboratory setup is shown in Fig. 5. We perform measurements for four different load settings; a first measurement with zero load (baseline), we next load it to a mid-load position corresponding to 5.5 Mpa, and then to full load position of $11.0 \mathrm{MPa}$, and finally a second measurement at zero load.

\section{RESULTS}

\subsection{Unloaded sample}

We first measure the scattered amplitudes for a sample under atmospheric conditions. The resulting out-of-plane and in-plane displacements are shown in Fig. 6. On the out-of-plane channel, the $P-P$ scattered wave arrives around $18 \mu \mathrm{s}$, followed by the converted $S V-P$ scattered event around $27 \mu$ s. The $P-P$ wave reflected from the back of the sample arrives at $37 \mu$ s, and the converted $S V-P$ reflection at $55 \mu \mathrm{s}$. The weaker unmarked events are side reflections and multiples. On the in-plane channel, an outgoing $S V$ phase, including the $P-S V$ scattering conversion, is visible at $27 \mu \mathrm{s}$, followed by the $S V-S V$ scattered wave at $36 \mu \mathrm{s}$, the $P-S V$ reflection from the back of the sample at $55 \mu \mathrm{s}$ and finally the $S V-S V$ reflection from the back of the sample at $73 \mu \mathrm{s}$. In order to extract the scattered amplitudes, we first bandpass the data around $1 \mathrm{MHz}$. This frequency corresponds to the maximum in energy generated by the thermoelastic source, but also to wavelengths $\lambda_{P}=2.6 \mathrm{~mm}$ and $\lambda_{S}=1.4 \mathrm{~mm}$. We are therefore in the single scattering regime described above, where the wavelength is in the order of the spatial extend of the fracture (radius $a=3.5 \mathrm{~mm}$ ). We then pick the maximum amplitudes for two events of interest: the $P$ wave scattered from an incoming $P$ wave, that is detected on the out-of-plane channel, and the $S V$ wave scattered from an incoming $S V$ wave that is 

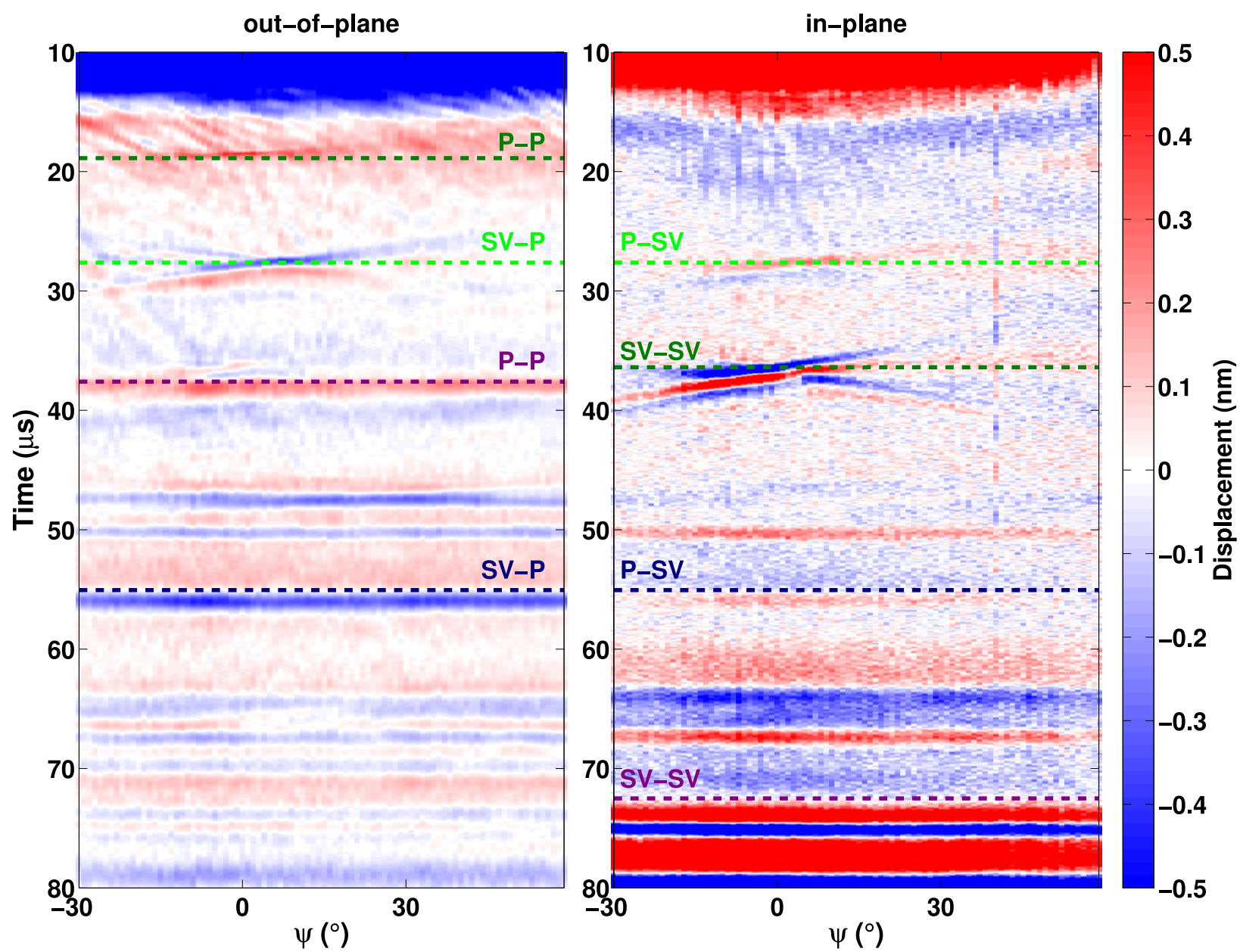

Figure 6. Displacement field for the unloaded sample, without filtering. Left: out-of-plane channel, right: in-plane channel. Both dark and light green dashed lines mark scattered arrivals, and light green marks converted ones. Purple dashed lines mark reflections from the backwall of the sample, and blue dashed lines mark mode conversions from the backwall.

detected on the in-plane channel. Both the scattered $P$ and scattered $S V$ amplitudes are normalized by the amplitude of the wave with the same mode reflected from the backwall of the sample, and corrected for geometrical spreading, effectively reducing the scattered amplitude to a fraction of the incoming amplitude.

For this geometry, the amplitude of the $P-P$ scattered is mostly sensitive to the normal component of the compliance $\eta_{N}$ (see eq. 1). Conversely, amplitude of the $S V-S V$ scattered event is mostly sensitive to the tangential component $\eta_{T}$ (eq. 3). We use a joint leastsquares regression to obtain the parameters giving the best fit with the experimental data, as well as the corresponding confidence intervals. We invert for the fracture radius $a$, the normal and tangential compliance and the orientation of the fracture, given by the angle $\theta_{0}$ between the normal to the fracture assumed before the start of the experiment, and the normal to the fracture obtained after inversion. This last parameter does not vary significantly from one measurement to another. The measured amplitudes and corresponding fits are shown in Fig. 7. The covariance matrix resulting from the inversion is shown in Fig. 8.

\subsection{Loading and unloading of the sample}

Next, we repeat the measurements described previously, but as a function of loading, in order to investigate the change in fracture

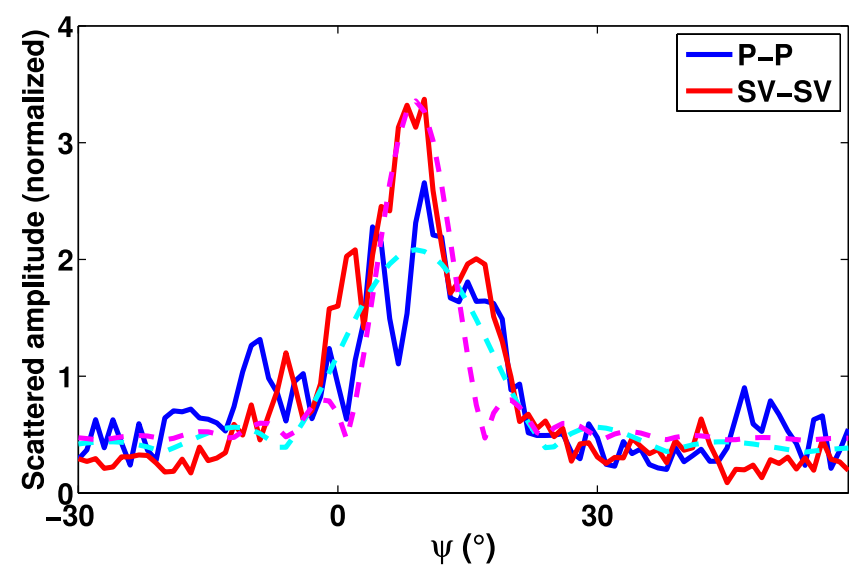

Figure 7. Measured (solid lines) and fitted amplitudes (dashed lines) for the $P-P$ and $S V-S V$ scattered events with an unloaded sample. From the fit, we get $a=3.14 \pm 0.19 \mathrm{~mm}, \eta_{N}=1.38 \pm 0.20 \cdot 10^{-11} \mathrm{~m} \mathrm{~Pa}^{-1}$ and $\eta_{T}=2.69 \pm$ $0.34 \cdot 10^{-11} \mathrm{~m} \mathrm{~Pa}^{-1}$.

properties with increasing stress. The measured amplitudes and corresponding fits for the maximum load measurement are shown in Fig. 9. A similar inversion scheme for the unloaded sample leads to estimates of the fracture-defining parameters. Then, we repeated the 


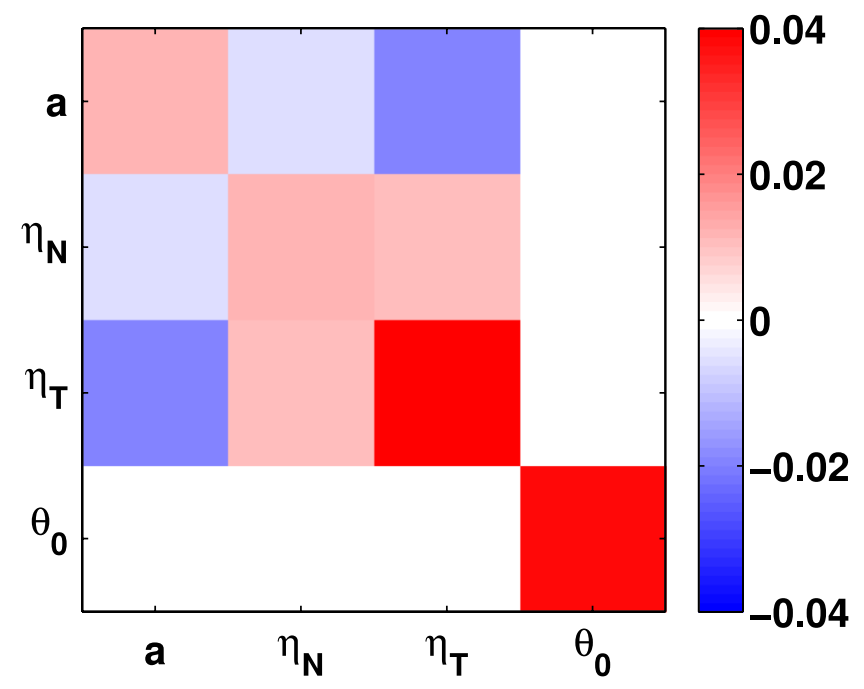

Figure 8. Covariance matrix resulting from the least-square inversion of the unloaded scattering data. This matrix is computed with the parameters expressed in units so that their values are between 1 and 10, with $a$ in millimetres, the compliances in $10^{-11} \mathrm{~m} \mathrm{~Pa}^{-1}$ and $\theta_{0}$ in degrees.

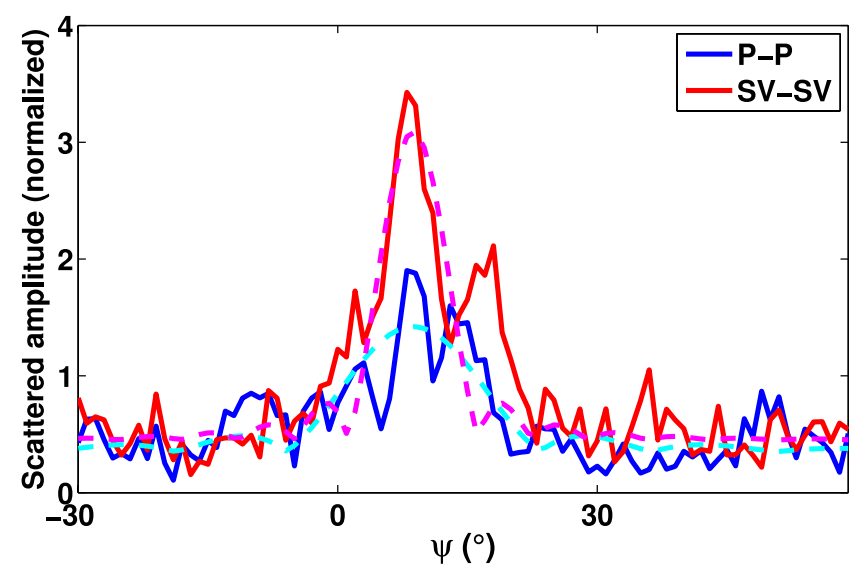

Figure 9. Measured (solid lines) and fitted amplitudes (dashed lines) for the $P-P$ and $S V-S V$ scattered events with a loaded sample at $11.0 \mathrm{MPa}$. From the fit, we get $a=3.32 \pm 0.22 \mathrm{~mm}, \eta_{N}=0.77 \pm 0.14 \cdot 10^{-11} \mathrm{~m} \mathrm{~Pa}^{-1}$ and $\eta_{T}=2.14 \pm 0.29 \cdot 10^{-11} \mathrm{~m} \mathrm{~Pa}^{-1}$.

process for intermediate loading values, and checked for hysteresis by repeating the no-load experiment after the loading sequence. The estimated parameters as a function of load are shown in Fig. 10 and Table 1 . We observe that the normal compliance $\eta_{N}$ decreases with increasing load. After the loading cycle, the estimated compliance is not exactly equal to the baseline value, but it is still higher than for the loaded case. The $P-P$ and $S V-S V$ backwall reflections stay constant in time for each loading stage, ruling out changes in the elastic properties of the homogeneous material.

\section{DISCUSSION}

The experimentally obtained $P-P$-wave scattering amplitudes are in good agreement with the theory of Blum et al. (2011), even though the geometry of the experiments differs. On the other hand, the results presented here involve a fracture with a spatial extend on the order of the elastic wavelength, which is of importance for exploration geophysics, where the size of fractures in the reservoir

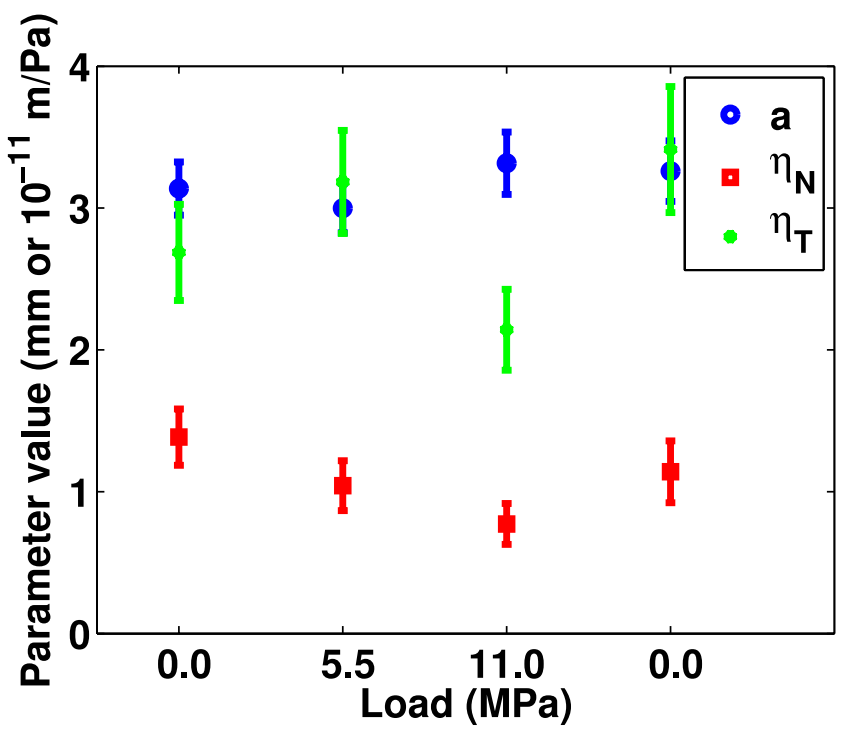

Figure 10. Estimates of the fracture radius $a$ (in blue) and the normal and tangential compliances, $\eta_{N}$ (in red) and $\eta_{T}$ (in green), respectively, during the loading cycle. The error bars correspond to the 95 per cent confidence intervals from the least-square fit.

is on the order of the seismic wavelength. Since the linear slip model has been verified experimentally for both natural (PyrakNolte et al. 1990a,b) and simulated fractures (Hsu \& Schoenberg 1993), we expect the results shown here to also be valid for rock fractures.

The estimated components of the compliance $\eta_{N}$ and $\eta_{T}$ are in the same order of magnitude. Moreover, by recording the $S V-S V$ scattering event, we are able to estimate $\eta_{T}$, and observe that $\eta_{N} / \eta_{T} \sim 0.5$. Such a ratio is also noted in other studies (Worthington 2007; Lubbe et al. 2008). The covariance matrix computed from the theoretical expressions indicates that the two components of the compliance are negatively correlated to the fracture radius, and the estimated values represent a trade-off between compliance and radius. The joint inversion ensures that the estimate of the radius estimate is consistent for both $P-P$ and $S V-S V$ data sets.

As mentioned above, the reflection and scattering traveltimes are constant with the increase in load, and show that the mechanical properties of the sample in the plane of the measurement do not change with the load. We confirm this by also measuring the amplitude of the reflections from the back of the cylinder at zero load and maximum load. The resulting measurements in Fig. 11 do not show a significant change between the two states of stress, for either the reflected $P$ and $S V$ waves. There is, however, an increase in $P P$ amplitude between $0^{\circ}$ and $10^{\circ}$, corresponding to the forward scattered wave, but independent of stress. The higher variability of the measured reflected SVSV amplitude precludes us from making a similar observation for this mode.

The static uniaxial load experiments show that the estimated radius is nearly constant over the cycle of four measurements. We conclude that the effective area (area of the fracture where a discontinuity is present in the material) of the fracture does not change for such stresses, as the load is too small to modify the structure of the fracture. We observe a decrease in normal compliance $\eta_{N}$ as the stress increases, and the final value of the normal compliance after the loading is not significantly lower than the value before the loading. This effect could be due to a small permanent plastic change of the fracture caused by the uniaxial stress. 
Table 1. Estimates of the fracture parameters with loading from the least-squares fit, with 95 per cent confidence intervals.

\begin{tabular}{lcccc}
\hline Load (MPa) & Radius $a(\mathrm{~mm})$ & $\eta_{N}\left(10^{-11} \mathrm{~m} \mathrm{~Pa}^{-1}\right)$ & $\eta_{T}\left(10^{-11} \mathrm{~m} \mathrm{~Pa}^{-1}\right)$ & $\theta_{0}\left(^{\circ}\right)$ \\
\hline 0.00 (initial) & $3.14 \pm 0.19$ & $1.38 \pm 0.20$ & $2.69 \pm 0.34$ & $1.0 \pm 0.4$ \\
0.55 & $3.00 \pm 0.17$ & $1.04 \pm 0.18$ & $3.18 \pm 0.36$ & $1.6 \pm 0.4$ \\
1.10 & $3.32 \pm 0.22$ & $0.77 \pm 0.14$ & $2.14 \pm 0.29$ & $1.3 \pm 0.4$ \\
0.00 (final) & $3.26 \pm 0.21$ & $1.14 \pm 0.22$ & $3.41 \pm 0.44$ & $1.1 \pm 0.4$ \\
\hline
\end{tabular}

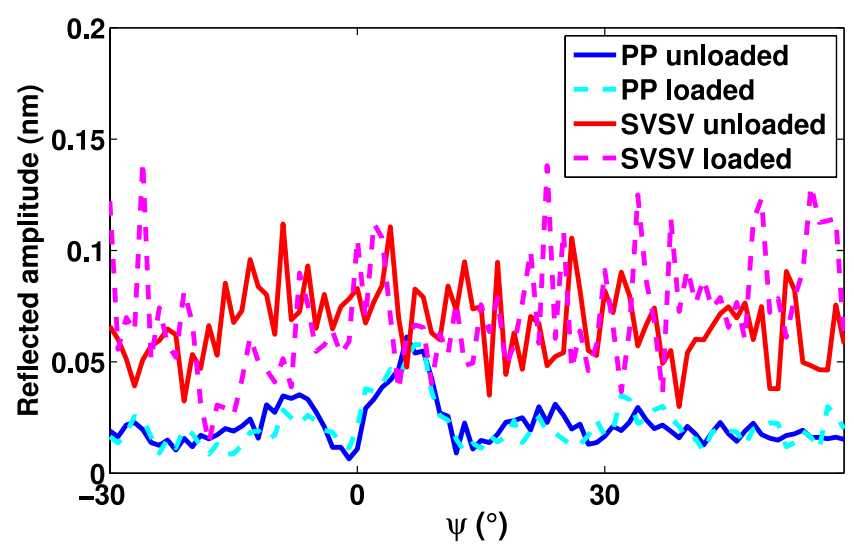

Figure 11. Amplitudes of the sample backwall reflection, at zero and maximum stress $(11.0 \mathrm{MPa})$, after filtering around $1 \mathrm{MHz}$. While there is no significant change introduced by the uniaxial stress, we observe a maximum in the reflected $P P$ amplitude at angles between $0^{\circ}$ and $10^{\circ}$, corresponding to the forward-scattering direction.

Most published laboratory studies of fractures involve uniaxial stresses normal to the fracture, and lead to a decrease in compliance with increasing load (Pyrak-Nolte et al. 1990a), which can be explained by the fracture becoming stiffer as the stress helps 'closing' it, and therefore increases the contact area of the fracture. Here, the stress axis is such that we would expect an opening of the fracture instead, leading to an increase in compliance. For bigger openings, however, the stiffness of round pores is greater than of elongated pores that more closely resemble natural fractures (Brie et al. 1985; Saleh \& Castagna 2004). By applying a load along a direction parallel to the fracture plane, the shape of the fracture changes from the ideal representation of a planar crack to a more rounded 3-D shape. Although this effect is small for the static load considered here, the observed change in compliance is consistent with a change in fracture shape from planar to more round-like.

Finally, it is much harder to interpret the tangential compliance estimates, compared to its normal component. The tangential values rely on the in-plane component of the wavefield. This measurement is much more sensitive to the positioning of the sample with respect to the laser receiver than the out-of-plane component. As we mechanically increase the stress on the sample, small changes in position lead to a bias - in addition to the data variance - on the in-plane wavefield recordings, as described in detail in Blum et al. (2010). The error bars shown in Fig. 10 do not encompass the (unknown) bias. Therefore, we cannot conclude that there are significant variations in the tangential component under the loads applied. Our results do show that by combining measurements of two components of the displacement field, we are able to estimate the size as well as the normal and tangential compliance of the fracture, paving the way for measurements under confining pressure (i.e. in situ subsurface conditions).

\section{CONCLUSION}

Because of the importance of fractures in geological processes, the full characterization of their elastic properties is critical to better understand the behaviour of fractures under stress. We show that the scattered amplitude of a plane fracture is in agreement with previously derived analytic expressions based on the linear slip model, for both the $P$ to $P$ and $S V$ to $S V$ scattering modes. Furthermore, the combined measurement of both scattered amplitudes allows us to estimate the size, orientation, as well as the normal and tangential components of the fracture compliance.

Moreover, we also look at the effects of static stress on fracture properties by applying uniaxial load parallel to the fracture plane. We monitor the fracture properties for four successive stages of stress and observe a significant decrease of the fracture normal compliance with increasing stress. We attribute this as a stiffening of the opening of a fracture. We are, however, unable to identify changes to the tangential compliance as a function of loading, due to limitations in the resolution of the in-plane wavefield detection.

\section{ACKNOWLEDGEMENTS}

We thank Randy Nuxoll for his help with the design and construction of the sample loader, and ConocoPhillips for financial support of this research.

\section{REFERENCES}

Blum, T.E., Snieder, R., van Wijk, K. \& Willis, M.E., 2011. Theory and laboratory experiments of elastic wave scattering by dry planar fractures, J. geophys. Res., 116, B08218, doi:10.1029/2011JB008295.

Blum, T.E., van Wijk, K., Pouet, B. \& Wartelle, A., 2010. Multicomponent wavefield characterization with a novel scanning laser interferometer, Rev. Sci. Instrum., 81(7), 073101.

Brie, A., Nurmi, R.D. \& Johnson, D.L., 1985. Effect of spherical pores on sonic and resistivity measurements, in Proceedings of 26th Annual Logging Symposium, no. Paper W, Society of Petrophysicists and WellLog Analysts.

Crampin, S., 1981. A review of wave motion in anisotropic and cracked elastic media, Wave Motion, 3, 343-391.

Gudmundsson, A., 2006. How local stresses control magma-chamber ruptures, dyke injections, and eruptions in composite volcanoes, Earth Sci. Rev., 79(1-2), 1-31.

Hsu, C.-J. \& Schoenberg, M., 1993. Elastic waves through a simulated fractured medium, Geophysics, 58(7), 964-977.

Hudson, J.A., 1981. Wave speeds and attenuation of elastic waves in materials containing cracks, Geophys. J. R. astr. Soc., 64, 133-150.

Hudson, J.A., Liu, E. \& Crampin, S., 1997. The mean transmission properties of a fault with imperfect facial contact, Geophys. J. Int., 129(3), 720-726.

Kachanov, M. \& Sevostianov, I., 2005. On quantitative characterization of microstructures and effective properties, Int. J. Solids Struct., 42, 309336.

Liu, E., Hudson, J.A. \& Pointer, T., 2000. Equivalent medium representation of fractured rock, J. geophys. Res., 105(B2), 2981-3000. 
Lubbe, R., Sothcott, J., Worthington, M. \& McCann, C., 2008. Laboratory estimates of normal and shear fracture compliance, Geophys. Prospect., 56(2), 239-247.

Pyrak-Nolte, L.J. \& Nolte, D.D., 1992. Frequency dependence of fracture stiffness, Geophys. Res. Lett., 19, 325-328.

Pyrak-Nolte, L.J., Myer, L.R. \& Cook, N.G.W., 1990a. Transmission of seismic waves across a single natural fracture, J. geophys. Res., 95(B6), 8617-8638.

Pyrak-Nolte, L.J., Myer, L.R. \& Cook, N.G.W., 1990b. Anisotropy in seismic velocities and amplitudes from multiple parallel fractures, J. geophys. Res., 95(B7), 11 345-11 358.

Saleh, A.A. \& Castagna, J.P., 2004. Revisiting the Wyllie time average equation in the case of near-spherical pores, Geophysics, 69(1), 45-55.

Schoenberg, M.A., 1980. Elastic wave behavior across linear slip interfaces, J. acoust. Soc. Am., 68, 1516-1521.
Schoenberg, M.A. \& Douma, J., 1988. Elastic wave propagation in media with parallel fractures and aligned cracks, Geophys. Prospect., 36, 571590.

Schoenberg, M.A. \& Sayers, C.M., 1995. Seismic anisotropy of fractured rock, Geophysics, 60, 204-211.

Scruby, C.B. \& Drain, L.E., 1990. Laser Ultrasonics Techniques and Applications, 1 st edn, Taylor \& Francis.

Worthington, M., 2007. The compliance of macrofractures, Leading Edge, 26, 1118-1121.

Zadler, B.J. \& Scales, J.A., 2008. Monitoring crack-induced changes in elasticity with resonant spectroscopy, J. appl. Phys., 104(2), 023 536-1023 536-4.

Zhu, Y. \& Snieder, R., 2002. Reflected and transmitted waves from fault zones, in Proceedings of SEG Technical Program Expanded Abstracts, vol. 21, pp. 2273-2276. 